CERN-ECP/95-26

7 November 1995

\title{
PION-INDUCED DAMAGE IN SILICON DETECTORS
}

\author{
S.J. Bates, C. Furetta, M. Glaser, F. Lemeilleur, E. León-Florián \\ CERN, 1211 Geneva 23, Switzerland \\ C. Gößling, B. Kaiser, A. Rolf, R. Wunstorf \\ Universität Dortmund, Exp. Physik IV, D-44221 Dortmund \\ H. Feick, E. Fretwurst, G. Lindström, M. Moll \\ Universität Hamburg, I. Institut für Experimentalphysik, Jungiusstraße 9, \\ D-20355 Hamburg \\ G. Taylor \\ Research Centre for High Energy Physics, University of Melbourne, \\ Parkville, Victoria 3052, Australia \\ A. Chilingarov \\ Budker Institute for Nuclear Physics, 630090 Novosibirsk, Russia
}

RD2 Collaboration

\begin{abstract}
The damage induced by pions in silicon detectors is studied for positive and negative pions for fluences up to $10^{14} \mathrm{~cm}^{-2}$ and $10^{13} \mathrm{~cm}^{-2}$, respectively. Results on the energy dependence of the damage in the region of $65-330 \mathrm{MeV}$ near to the $\Delta$ resonance are presented. The change in detector characteristics such as leakage current, charge collection efficiency and effective impurity concentration including long-term annealing effects have been studied. Comparisons to neutron- and proton-induced damage are presented and discussed.
\end{abstract}

(Submitted to Nuclear Instruments and Methods in Physics Research) 



\section{INTRODUCTION}

Silicon detectors used in future Large Hadron Collider (LHC) experiments will be exposed to huge fluxes of various particle types. In the past many studies on irradiation damage have been carried out especially with neutrons and protons (see [1] and references therein). The change of diode reverse current, full depletion voltage and charge collection efficiency has been characterized as a function of the particle fluence and energy.

Recent calculations of particle fluences to be expected in LHC experiments show thatespecially in the barrel region up to $40 \mathrm{~cm}$ from the beam axis-pions represent the major contribution to the total particle flux [2], [3]. Most of the pions have kinetic energies between $50 \mathrm{MeV}$ and $1000 \mathrm{MeV}$, the peak of the energy distribution is around $250 \mathrm{MeV}$, near to the $\Delta$ resonance. These results underline the necessity of performing pion irradiations in order to compare the radiation damage due to pions with those results obtained for neutrons and protons. Furthermore, one must investigate whether the larger $\pi$-nucleus total cross-section in the $\Delta$ resonance region leads to enhanced damage in silicon detectors.

Irradiations have been carried out by several groups of the RD2 Collaboration at the highflux pion beam line at the Paul Scherrer Institute (PSI) in Villigen, Switzerland. The experimental programme included a high fluence $\pi^{+}$irradiation (up to $10^{14} \mathrm{~cm}^{-2}$ ) with a momentum of $350 \mathrm{MeV} / c$, low fluence $\pi^{+}$irradiations at various pion momenta, and irradiations with negative pions.

This paper presents results on electrical characteristics and charge collection efficiency obtained by the various groups for different detectors using independent measurement setups.

\section{EXPERIMENTAL DETAILS}

All pion irradiations were carried out at the high-flux pion beam line $\pi \mathrm{E} 1$ at the Paul Scherrer Institute. Details of beam line setup, beam control, and beam contamination measurements can be found in Ref. [4]. Pions are produced by a collision of protons coming from a cyclotron of up to $800 \mu \mathrm{A}$ beam current with a graphite target. They are transferred by a beam line containing a magnet spectrometer in order to select pions according to charge and momentum. Behind this beam line, carbon plates of appropriate thickness are used as absorbers for the protons and antiprotons accompanying the pion beam. Pion fluxes are dependent on energy; the highest flux of approximately $7 \times 10^{8} \mathrm{~cm}^{-2} \mathrm{~s}^{-1}$ is reached for $350 \mathrm{MeV} / c$ positive pions, negative pion fluxes are about a factor 10 lower than those for positive pions. The beam contamination by neutrons was measured to be less than $1 \%$ of the pion flux.

The beam position and its transverse and lateral profile was measured using an XY chamber which could be moved along the beam axis. An ionization chamber was permanently placed in the beam in order to record the flux-time profile during the irradiations. The pion fluence measurements were performed using the activation of aluminium foils. Several foils were put in the stack of detectors which was placed in the approximately $30 \mathrm{~cm}$ long irradiation zone. After each irradiation step the $\gamma$ activity of each foil due to the ${ }^{27} \mathrm{Al}\left(\pi^{ \pm}, \mathrm{xN}\right)^{24} \mathrm{Na}$ reaction was measured by a $\gamma$ spectrometer. The result was used to calculate the pion fluence, knowing the reaction cross-section and the flux-time profile. The fluence error is of the order of $\pm 5 \%$. Details of this procedure are described in Refs. [4] and [5]. 
Several different detector types described in Table 1 have been used for these studies. This enables us to check whether the irradiation damage is dependent on the detector type or manufacturer; however, the origin of the silicon wafers is almost entirely Wacker. All detectors were manufactured from n-type silicon.

Table 1

List of detectors used by the different groups. (Do: Dortmund University; ECP: CERN ECP division; HH:

Hamburg University; PPE: CERN PPE division.) The segmented detectors used by PPE consist of 64 pads arranged in an $8 \times 8$ pattern. (Wacker: Wacker Chemietronics, Burghausen, Germany; MPI: MPI Semiconductor Laboratory, München, Germany; MSU: Moscow State University, Moscow, Russia; Micron: Micron Semiconductor Ltd., UK; Intertechnique: Intertechnique Groupe, Plaisir, France.)

\begin{tabular}{|c|c|c|c|c|c|c|}
\hline Group & $\begin{array}{c}\text { Detector } \\
\text { type }\end{array}$ & $\begin{array}{c}\text { Single/ } \\
\text { segmented }\end{array}$ & $\begin{array}{c}\text { Area } \\
\left(\mathrm{mm}^{2}\right)\end{array}$ & $\begin{array}{c}\text { Thickness } \\
(\mu \mathrm{m})\end{array}$ & $\begin{array}{c}\text { Wafer } \\
\text { material }\end{array}$ & Manufacturer \\
\hline Do1 & ion-impl. & single & $5 \times 5$ & 280 & Wacker & MPI \\
Do2 & ion-impl. & single & $\begin{array}{c}10 \times 10 \\
\text { Tesla/Wacker }\end{array}$ & MSU \\
\hline ECP & ion-impl. & single & $\begin{array}{c}5 \times 5, \\
10 \times 10\end{array}$ & 300 & Wacker & Micron \\
\hline HH1 & surface-barrier & single & 16 & 390 & Wacker & HH \\
HH2 & ion-impl. & single & $5 \times 5$ & 300 & Wacker & Micron \\
\hline PPE & ion-impl. & segmented & $\begin{array}{c}3 \times 3 \\
\text { per pad }\end{array}$ & $305 \pm 10$ & Wacker & $\begin{array}{c}\text { Micron/ } \\
\text { Intertechnique }\end{array}$ \\
\hline
\end{tabular}

\section{RESULTS}

\subsection{Energy dependence of pion displacement function}

The non-ionizing energy loss (NIEL) for pions in the energy region of 100-600 MeV has been estimated by Aarnio and Huhtinen [3]. This calculation predicts that the NIEL and therefore the expected irradiation damage is in the order of the NIEL for $1 \mathrm{MeV}$ neutrons and shows a slight increase in the region of the $\Delta$ resonance. In order to check that prediction, an energy scan was performed for fluences up to about $10^{12} \mathrm{~cm}^{-2}$. The change in the effective impurity concentration $\mathrm{N}_{\text {eff }}$ for such small fluences is dominated by donor removal with unknown starting concentrations. Therefore it is impossible to determine either the donor removal rate or the acceptor generation rate out of $\mathrm{N}_{\text {eff }}$ measurements. Therefore the current increase - which was up to now always found to be proportional to the fluence-was taken as a measure for the energy-dependent defect generation rate which should be proportional to the NIEL.

In order to keep the comparison between different groups simple, the current damage constant $\alpha_{\infty}$ is quoted here. This is determined by the part of the volume current increase $\Delta \mathrm{I} / \mathrm{Vol}$ which does not anneal even after very long observation periods (for details of the annealing behaviour see Section 3.2): 


$$
\alpha_{\infty}=\frac{\Delta \mathrm{I}\left(\mathrm{V}=\mathrm{V}_{\mathrm{depl}}, \mathrm{t} \rightarrow \infty\right)}{\Phi \times \mathrm{Vol}}
$$

where the current is measured for the voltage of full depletion $\left(\mathrm{V}_{\mathrm{depl}}\right), \Phi$ denotes the pion fluence and $\mathrm{Vol}$ the active detector volume. All currents are scaled to $20^{\circ} \mathrm{C}$ using the equation

$$
\mathrm{I} \propto \mathrm{T}^{2} \times e^{-\mathrm{E}_{\mathrm{g}} / 2 \mathrm{kT}}
$$

where $\mathrm{E}_{\mathrm{g}}$ is the silicon band gap energy, $\mathrm{T}$ the temperature and $\mathrm{k}$ the Boltzmann constant. Table 2 lists the results which were directly taken out of measurements made more than nine months after irradiation except for the $\mathrm{HH}$ data, where $\alpha_{\infty}$ was calculated from data taken earlier and applying the current annealing function from Ref. [6]. Figure 1 shows $\alpha_{\infty}$ versus the kinetic energy of the pions. For comparison the prediction of Ref. [3] has been normalized to the data and plotted as a solid line on the same figure.

Unfortunately the error bars and the variation from detector to detector are of the order of the expected NIEL variation within the examined energy region. This means that one can only see weak evidence for the expected behaviour around the $\Delta$ resonance. Nevertheless, one can exclude that the $\Delta$ resonance enhancement of pion radiation damage is noticeably stronger than the one predicted by calculations [3].

\section{Table 2}

Current damage constant $\alpha_{\infty}$ for different pion energies. Values measured after the current annealing process and corrected to $20^{\circ} \mathrm{C}$ using eq. (2). HH data measured earlier and corrected (see text).

\begin{tabular}{|c|c|c|c|c|c|c|}
\cline { 3 - 7 } \multicolumn{2}{c|}{} & \multicolumn{5}{|c|}{$\alpha_{\infty}\left(10^{-17} \mathrm{~A} / \mathrm{cm}\right)$} \\
\hline $\begin{array}{c}\text { Pion } \\
\text { momentum } \\
(\mathrm{MeV} / c)\end{array}$ & $\begin{array}{c}\text { Kinetic } \\
\text { energy } \\
(\mathrm{MeV})\end{array}$ & Do1 & HH1 & HH2 & PPE & ECP \\
\hline 150 & 65 & & & $2.11 \pm 0.12$ & $1.7 \pm 0.3$ & $2.33 \pm 0.35$ \\
250 & 147 & $2.9 \pm 0.3$ & & $3.32 \pm 0.29$ & & $2.72 \pm 0.35$ \\
300 & 192 & & $3.24 \pm 0.19$ & $2.74 \pm 0.15$ & $2.9 \pm 0.2$ & $3.21 \pm 0.33$ \\
350 & 236 & $4.1 \pm 0.4$ & $2.74 \pm 0.19$ & $2.6 \pm 0.14$ & $2.6 \pm 0.2$ & $2.67 \pm 0.32$ \\
400 & 281 & $2.9 \pm 0.4$ & & $3.77 \pm 0.22$ & $2.2 \pm 0.1$ & $3.05 \pm 0.41$ \\
450 & 330 & $3.4 \pm 0.3$ & & & $2.6 \pm 0.3$ & $2.53 \pm 0.31$ \\
\hline
\end{tabular}




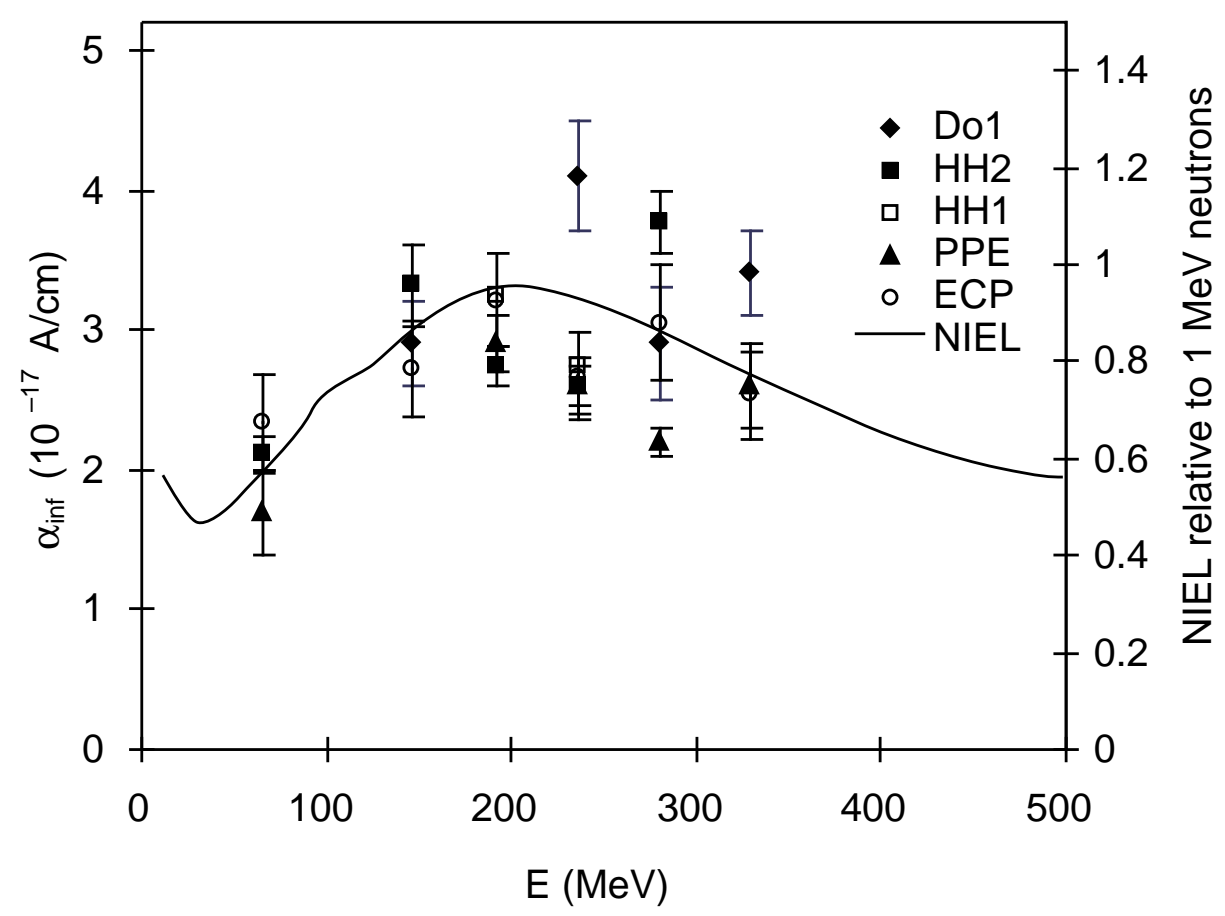

Fig. 1: Energy dependence of current damage constant $\alpha_{\infty}$ after complete annealing. Data corrected to $20{ }^{\circ} \mathrm{C}$.

\subsection{Short-term current annealing}

The short-term annealing behaviour of detector leakage currents has to be studied in order to correct for the annealing which occurs during the irradiations lasting up to a few days. These corrections allow a comparison of current damage results taken from irradiation experiments with different flux-time profiles. A single diode of the ECP type was irradiated in a $26 \mathrm{~min}$ long irradiation with $350 \mathrm{MeV} / c$ positive pions to a fluence of $(8.6 \pm 0.3) \times 10^{11} \mathrm{~cm}^{-2}$. The current was monitored for the subsequent two days at a bias voltage of $80 \mathrm{~V}$. The voltage for full depletion had evolved from $26 \mathrm{~V}$ to $20 \mathrm{~V}$ due to the irradiation. Thus the variation of the overbias was negligible. The detector was stored in the irradiation area in an ambient temperature of $25 \pm 1{ }^{\circ} \mathrm{C}$, and the current was normalized to $25^{\circ} \mathrm{C}$ using Eq. (2).

It is common practice to fit the annealing data to a sum of exponential decays having different amplitudes and time constants [6] including a constant term, which, in this case, is the part of the current that has not been annealed after the 48-hour observation period:

$$
\frac{\mathrm{I}\left(\mathrm{t}^{\prime}\right)}{\mathrm{I}_{\mathrm{T}}}=\sum_{\mathrm{i}=1}^{\mathrm{n}} \mathrm{A}_{\mathrm{i}}^{*} e^{-\mathrm{t}^{\prime} / \tau_{\mathrm{i}}}
$$

where $t^{\prime}$ is the time after the end of irradiation and $I_{T}$ the current directly after irradiation. Table 3 shows a list of the fit parameters $\mathrm{A}_{\mathrm{i}}^{*}$ and $\tau_{\mathrm{i}}$.

They are used to correct for self-annealing after irradiation to the current value $\mathrm{I}_{0,26 \mathrm{~min}}$, which would be measured if no annealing had taken place after the 26 min of irradiation [6], [7].

A comparison of the current annealing to the annealing behaviour observed after irradiation with neutrons is given in Fig. 2, where the current measured after irradiation is normalized to the stable fraction of the current $\mathrm{I}_{\infty}=\mathrm{I}(\mathrm{t} \rightarrow \infty)$. The squares indicate the ECP 
data points and the dashed line shows the fit using the values of Table 3 . The open circles show results from measurements of the PPE group on six of their detectors after longer annealing times. The relative scaling of ECP to PPE data was done by adjusting the values of the first three PPE measurement points to the ECP fit.

Table 3

Self-annealing parameters according to the fit function Eq. (3) for $350 \mathrm{MeV} / c$ pions $\left(\Phi=8.59 \times 10^{11} \mathrm{~cm}^{-2}\right.$, irradiation time $\left.26 \mathrm{~min}\right)$.

Observation period $48 \mathrm{~h}$ after end of irradiation.

\begin{tabular}{|c|c|}
\hline $\mathrm{A}_{\mathrm{i}}^{*}(26 \min )$ & $\tau_{\mathrm{i}}(\min )$ \\
\hline $0.175 \pm 0.007$ & $5.55 \pm 0.30$ \\
$0.110 \pm 0.005$ & $80 \pm 4$ \\
$0.183 \pm 0.005$ & $922 \pm 31$ \\
$0.574 \pm 0.030$ & $\infty$ \\
\hline
\end{tabular}

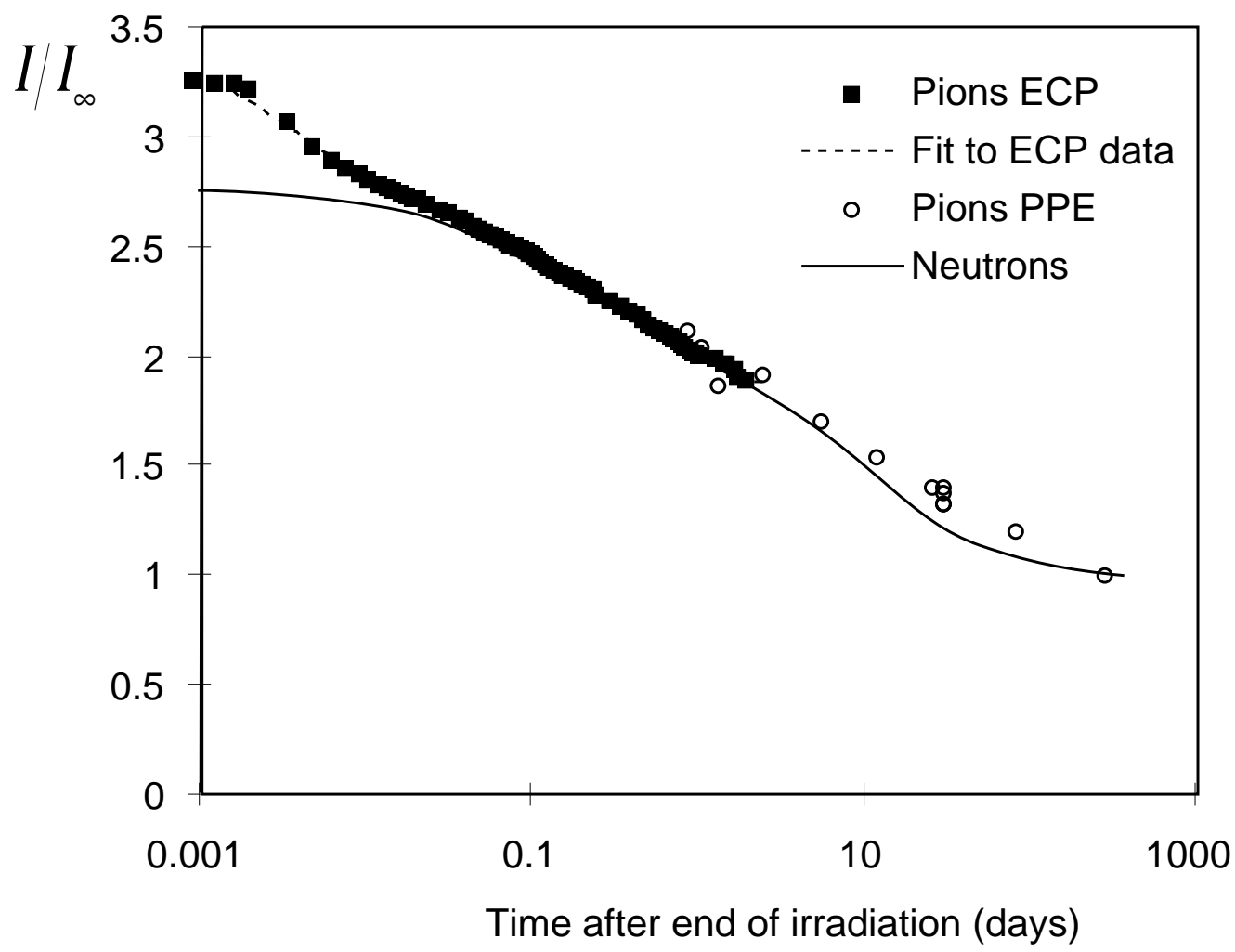

Fig. 2: Short-term current annealing for pions and neutrons following a short (26 min) irradiation. Pion data taken from measurements, neutron curve taken from Ref. [6], (data for detectors before inversion) and recalculated for $26 \mathrm{~min}$ irradiation time. 
The data for neutrons is taken from Ref. [6] where the annealing has been studied after 5 min irradiations. For the comparison shown in Fig. 2 this has been calculated as 26 min irradiation duration.

From the figure one can conclude that neutron and pion annealing obviously shows the same time dependence. There are only small differences for the very short-term annealing of up to two hours. These deviations are most probably a systematic error of the correction for the irradiation times. The measurements could depend differently on annealing processes with time constants comparable to the irradiation duration. One can nevertheless assume that the current damage due to both particle types is based on the same type and mixture of defects.

\subsection{Reverse current}

There are two possible ways to characterize the reverse current damage. The first is to quote $\alpha_{\text {corr,26min }}$ from $\mathrm{I}_{0,26 \mathrm{~min}}$ as the self-annealing corrected value using the correction method described in Ref. [7] and the parameters from Table 3. This method was carried out using detectors of the ECP and Do1 type, where the irradiation was done using very few detectors, irradiated in approximately ten fluence steps, and measured in the irradiation breaks.

The other possibility, in order to rule out annealing effects, is to quote the $\alpha_{\infty}$ value already defined in Section 3.1. This value is more significant for prediction of long-term operation currents in the LHC experiments.

Figure 3 contains the temperature- and self-annealing-corrected current $\mathrm{I}_{0,26 \mathrm{~min}}$ versus the fluence for negative and positive pions of $350 \mathrm{MeV} / \mathrm{c}$ momentum. The plot shows that the current depends linearly on the fluence in the whole fluence region examined. It furthermore shows good agreement of both detector types and no difference between positive and negative pions. A common fit to all data points results in $\alpha_{\text {corr, } 26 \min }=(9.4 \pm 0.2) \times 10^{-17} \mathrm{~A} / \mathrm{cm}$. Taking into account the NIEL prediction of Ref. [3] $\left(\kappa=0.93, \Phi_{\mathrm{eq}, 1 \mathrm{MeV}}=\kappa \times \Phi\right)$ this leads to a $1 \mathrm{MeV}$ neutron equivalent value of $\alpha_{\text {corr,26min,eq }}=(10.1 \pm 0.2) \times 10^{-17} \mathrm{~A} / \mathrm{cm}$. This is of the same order as measurements on neutron-irradiated detectors, where the results vary in the range of $(7-11) \times 10^{-17} \mathrm{~A} / \mathrm{cm}$ and the annealing correction procedure was carried out in a similar way relying on annealing measurements after 5 min irradiations. The large variation within the data for neutrons may be due not only to different detector types but may also be explained by uncertainties in the fluence normalization according to the energy and different self-annealing correction procedures [8].

Calculating an $\alpha_{\infty}$ value out of $\alpha_{\text {corr,26min }}$ by

$$
\alpha_{\infty}=\alpha_{\text {corr, } 26 \min } \times \frac{I(t=0)}{I_{\infty}}
$$

(see Section 3.2) one obtains $(3.04 \pm 0.07) \times 10^{-17} \mathrm{~A} / \mathrm{cm}$. This result can be compared with the direct measurements of $\alpha_{\infty}$ carried out by all groups as shown in the following paragraph. 


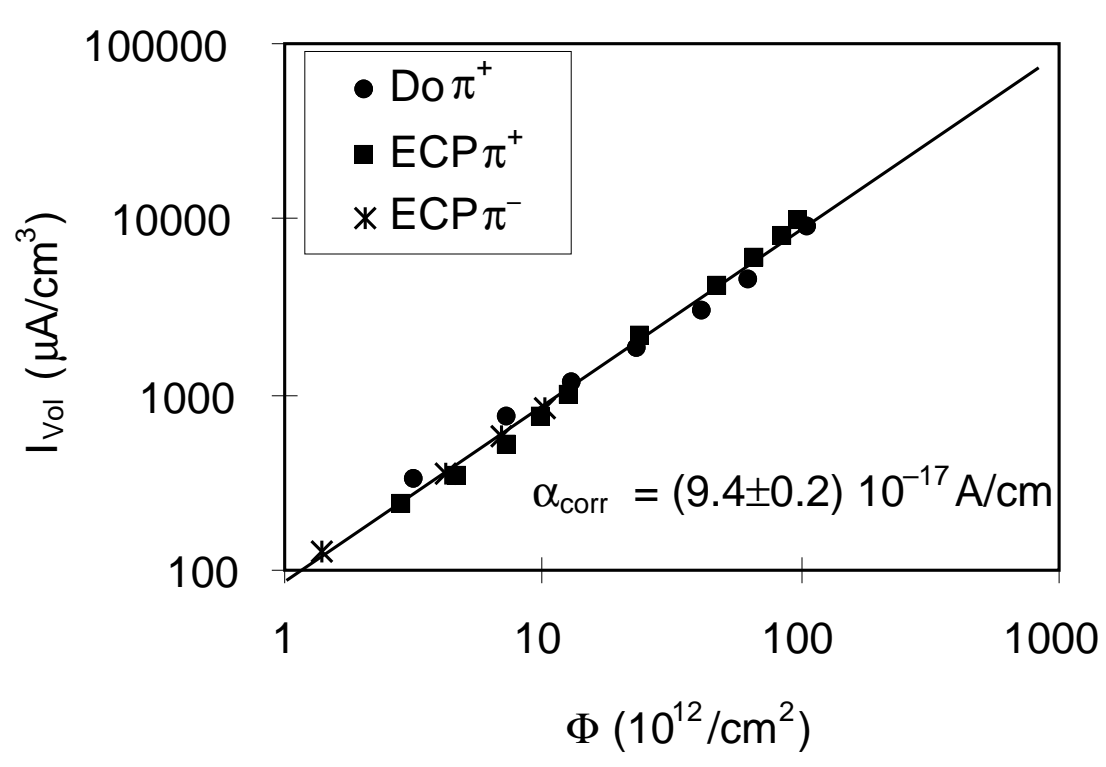

Fig. 3: Volume leakage current versus pion fluence. Temperature and self-annealing corrected according to 26 min irradiation duration. Pion momentum $350 \mathrm{MeV} / c$. Solid line is a fit to all points.

Figure 4 shows the $\alpha_{\infty}$ values as they were measured for the different detector types, where in this case each data point represents one individual detector after irradiation to the indicated fluence of $350 \mathrm{MeV} / c$ pions. Unfortunately it turns out to be difficult to quote an $\alpha_{\infty}$ value common to all detector types and fluences. For larger fluences $\alpha_{\infty}$ is no longer a constant but often shows higher values than expected. The following comments try to explain this observation:

- Do 2: The value $\alpha_{\infty}$ is slowly increasing for the three lowest fluences. The currentvoltage curves of all detectors show a further increase of the current for $\mathrm{V}>\mathrm{V}_{\text {depl }}$. This increase becomes stronger and stronger with fluence and finally for the detector with $\Phi=6.65 \times 10^{13} \mathrm{~cm}^{-2}$ the detector shows an exponentially growing current for $\mathrm{V}<\mathrm{V}_{\mathrm{depl}}$. This indicates that the current contributions dominating for large fluences are not due to volume effects. They can, for example, be due to regions of large electric fields at the detector surface and edges. The magnitude of these additional currents has been estimated for one detector by extrapolating the current curve before breakdown to $V_{\text {depl }}$. The result is indicated in Fig. 4.

- $\quad$ HH1: The $\alpha_{\infty}$ values for devices irradiated past inversion and having undergone an elevated temperature annealing at $50^{\circ} \mathrm{C}$ exhibit a large variation from detector to detector, probably not dependent on fluence, but being all significantly higher than the low fluence value. Since all the detectors used here come from the same wafer, variations from wafer to wafer can be excluded as an explanation. The following explanations might be applicable:

- Enhanced introduction of current generation centres after inversion. This has also been observed earlier for neutron-irradiated detectors of a very similar type [9].

- After inversion the rectifying contact is established by the aluminium-silicon interface, which is not well understood. 
- Large systematic errors concerning the depleted volume due to the unknown field propagation in inverted detectors.

- PPE: Only one measurement available which fits into the low fluence data of the other groups.

- $\quad$ ECP: The highly irradiated detector shows a strong breakdown-like behaviour occurring for bias voltage lower than $\mathrm{V}_{\mathrm{depl}}$. The same comment as for Do2 can be applied. The additional current is not annealing with time, one even observes an increase of the total leakage current with the 'annealing' time.

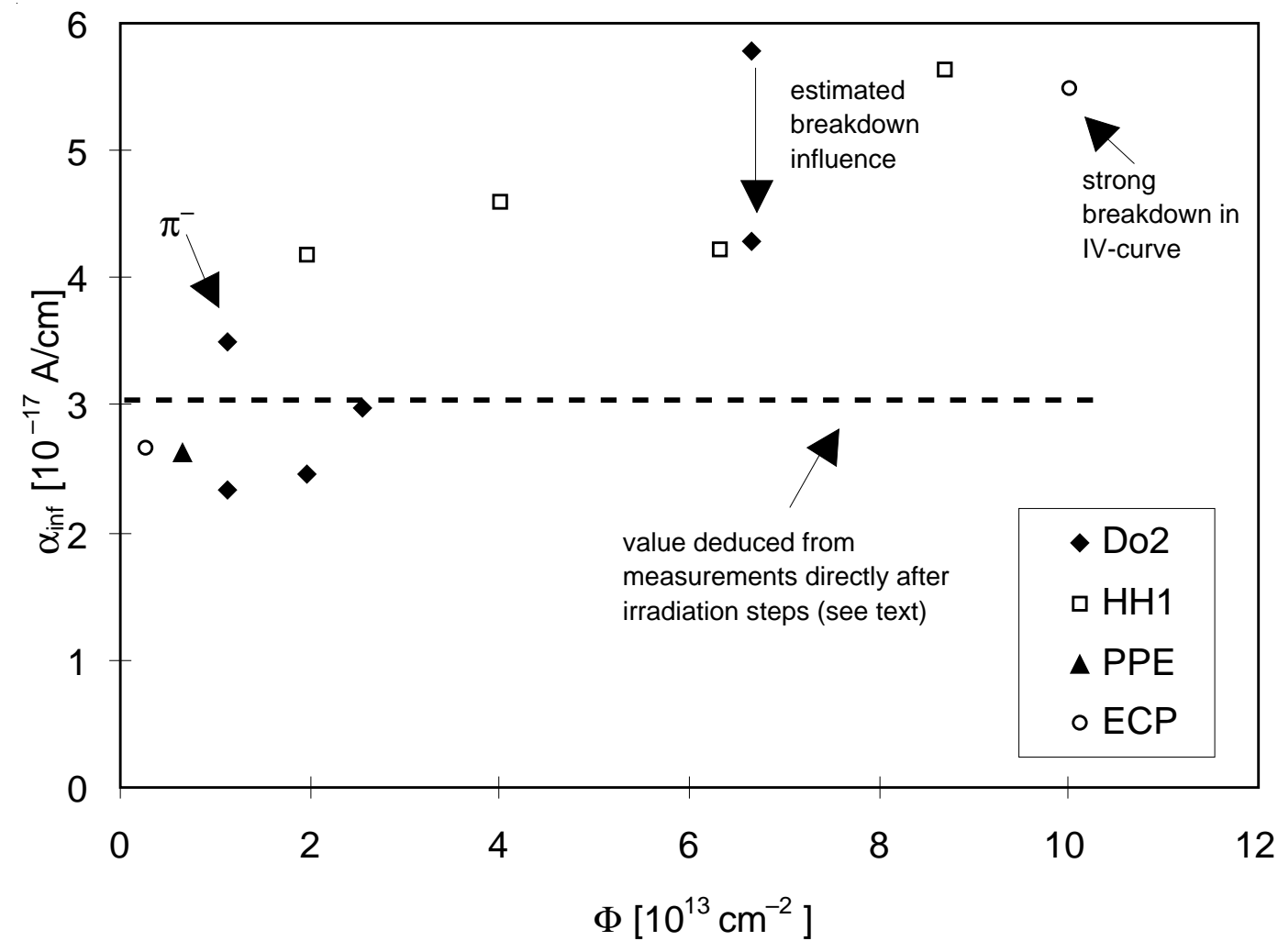

Fig. 4: Values of $\alpha_{\infty}$ for $350 \mathrm{MeV} / c$ pions as obtained by measurements on individual detectors and measured after at least nine months of annealing at room temperature, or elevated temperature annealing at $50^{\circ} \mathrm{C}$ in the case of $\mathrm{HH} 1$.

Considering all these comments, one may conclude the following: For low fluences the measured current damage constant $\alpha_{\infty}$ agrees with the value $\alpha_{\text {corr,26min }}$ quoted above and is therefore also consistent with the damage induced by neutrons. For larger fluences the current increase is no longer strictly proportional to the fluence, there are additional current contributions. These seem to be dependent on the detector type and show a different time dependence than the one introduced in the last section. Similar effects have also been observed earlier for irradiations with other particle types [9], [10] and large variations in leakage current at high bias voltages are seen for different detectors even before irradiation. They are probably a function of the processing. It is hoped that future detectors will have higher voltage tolerance so that after irradiation, when depletion voltages are high, they may be operated without the sharp 
increase in leakage current mentioned above. Thus the volume current due to irradiation by pions can be treated like that introduced by neutrons.

In order to give predictions of total detector currents in LHC experiments, the role of irradiation-induced surface currents has to be studied in greater detail including ageing effects in irradiated detectors.

\subsection{Charge collection efficiency}

The charge collection efficiency CCE was measured by two of the participating groups (ECP and PPE). It is defined as the charge collected for irradiated detectors $Q_{\text {irr }}$ normalized to the value $\mathrm{Q}_{0}$ measured before irradiation:

$$
\mathrm{CCE}=\frac{\mathrm{Q}_{\text {irr }}}{\mathrm{Q}_{0}} .
$$

Both measurements use relativistic $\beta$ particles from a radioactive source and a scintillation detector for triggering.

In the ECP setup the current pulse coming from a fast current preamplifier is directly investigated. All the charge is collected in less than $20 \mathrm{~ns}$, and the total charge is calculated by integrating this current signal. For details of the setup see Ref. [11]. Measurements were made at room temperature directly after each irradiation step except for the highest fluence where the data was taken 74 days after irradiation.

The PPE setup uses the fast charge sensitive BIPOLTEST preamplifier with a peaking time of about $15 \mathrm{~ns}$ [12]. The measurements after irradiation were taken at $0{ }^{\circ} \mathrm{C}$ in order to reduce the leakage current during the measurement. The temperature dependence of the CCE is discussed in Ref. [12] and is of minor importance compared for example with the strong dependence on the bias voltage.

For both measurements a minimum bias voltage of $100 \mathrm{~V}$ was applied for $\mathrm{V}_{\mathrm{depl}}<50 \mathrm{~V}$, in the other cases an overbias of about $50 \mathrm{~V}$ was used.

Figure 5 shows the results of the CCE measurements. For comparison the data for neutrons are included. These were calculated from the measured trapping time constants taking into account the applied bias voltage and its influence on the internal electric fields. For details of the calculation and the input parameters see Ref. [1].

One can see that the CCE is the same for both detector types and is also comparable to the values calculated for neutron damage. Thus the CCE loss due to pion irradiation appears to be no problem for the LHC application, especially since further measurements shown in Fig. 6 indicate that the charge loss can even be reduced by a moderate increase of the detector overbiasing. 


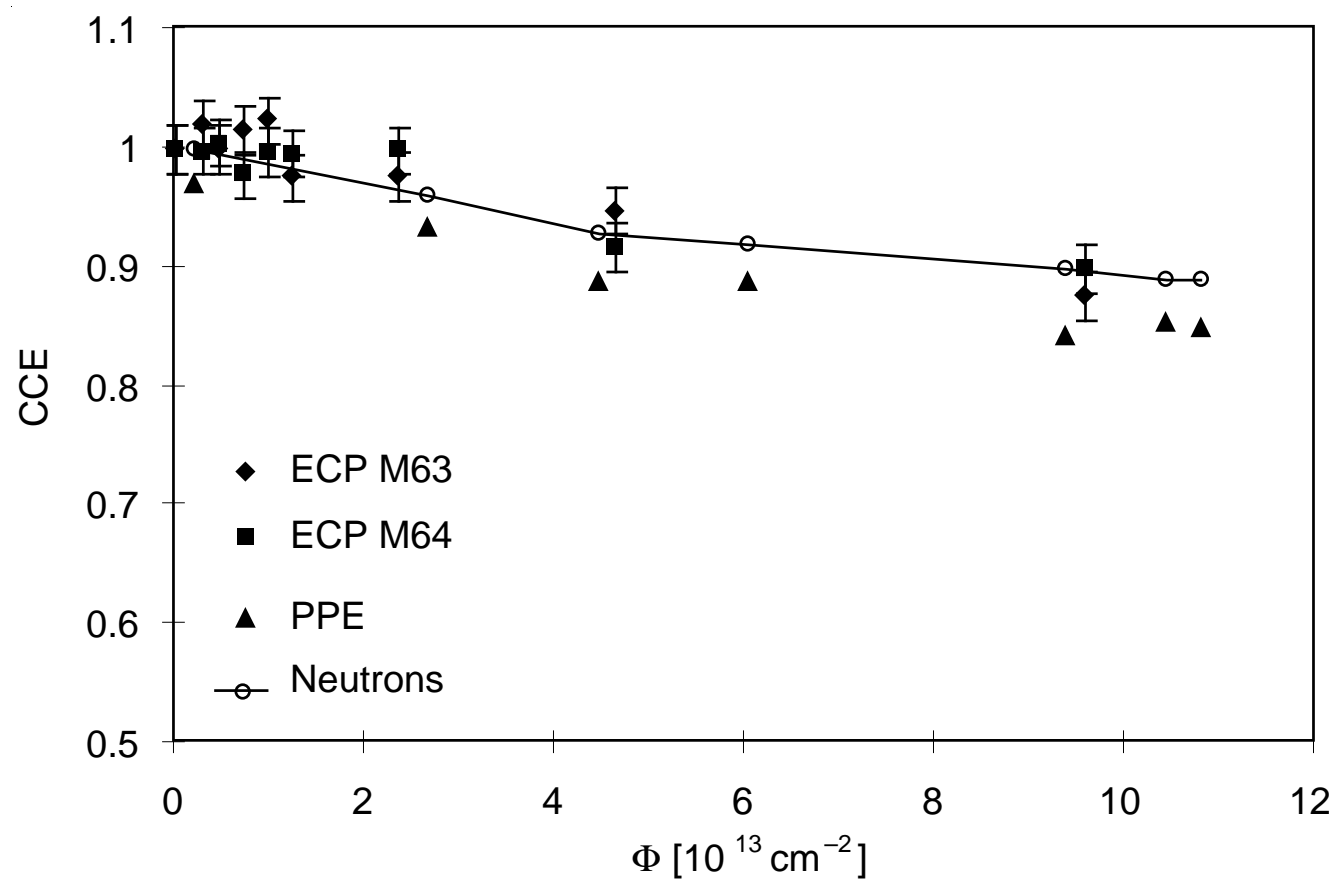

Fig. 5: Charge collection efficiency (CCE) for pion-irradiated detectors. M63, M64: ECP-type detectors, PPE: PPE-type detectors, each data entry refers to one detector. For a description of the measurement details see text. Neutron CCE calculated according to the Hamburg group simulation model [1] for comparison taking into account the biasing values used in the measurements.

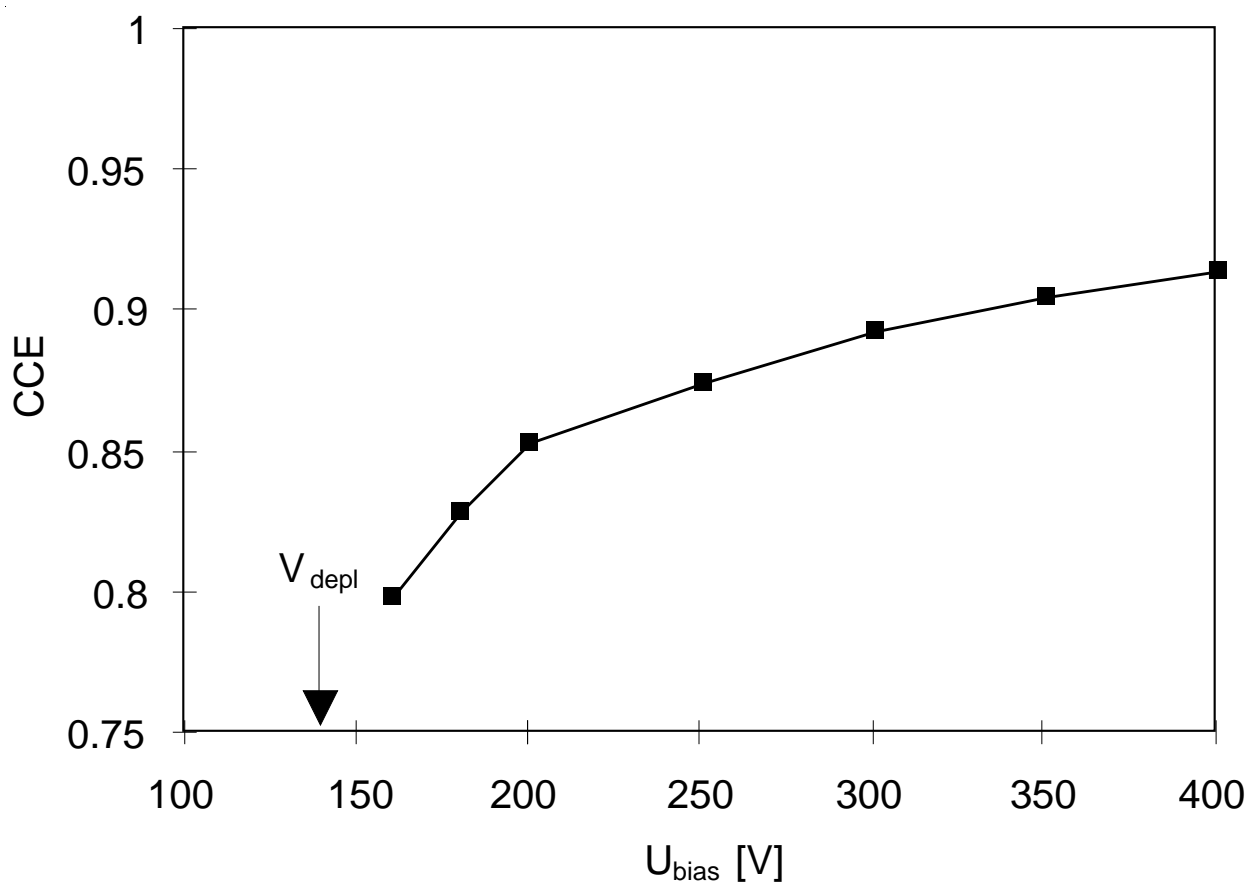

Fig. 6: Example for the dependency of the charge collection efficiency on the bias. PPE-type detector, $\Phi=1.04 \times 10^{14} \mathrm{~cm}^{-2}, \mathrm{~V}_{\mathrm{depl}}=37 \mathrm{~V}$, measured at $0{ }^{\circ} \mathrm{C}$. 


\subsection{Effective impurity concentration}

The absolute value of the effective impurity concentration $\mathrm{N}_{\text {eff }}$ is determined from the full depletion voltage $\mathrm{V}_{\mathrm{depl}}$ using the following equation:

$$
\left|\mathrm{N}_{\mathrm{eff}}\right|=\left(\mathrm{V}_{\mathrm{depl}}+\mathrm{V}_{\mathrm{bi}}\right) \times \frac{2 \varepsilon_{\mathrm{r}} \varepsilon_{0}}{\mathrm{ed}^{2}}
$$

where $\mathrm{V}_{\mathrm{bi}}$ is the junction built-in voltage $(\approx 0.6 \mathrm{~V}), \varepsilon_{\mathrm{r}}$ the relative permittivity of silicon, $\varepsilon_{0}$ the vacuum permittivity, e the electron charge and $d$ the thickness of the detector. The values $\mathrm{V}_{\text {depl }}$ and $\mathrm{N}_{\text {eff }}$ can be determined from capacitance-voltage characteristics even after high fluences [6].

The fluence dependence of $\mathrm{N}_{\text {eff }}$ without any annealing processes taken into account has been shown to have the following form for neutrons and protons [6], [11]:

$$
\left|\Delta \mathrm{N}_{\mathrm{eff}}(\Phi)\right|=\left|\mathrm{N}_{\mathrm{eff}}(\Phi)-\mathrm{N}_{\mathrm{eff}, 0}\right|=\left|\mathrm{N}_{\mathrm{D}}\left(e^{-\mathrm{c} \Phi}-1\right)-\mathrm{b} \Phi\right|
$$

where $\mathrm{N}_{\mathrm{D}}$ is the initial donor concentration, $\mathrm{c}$ is a measure for the donor removal, and $\mathrm{b}$ is the rate of acceptor creation, which is the dominating process for fluences larger than approximately $10^{13} \mathrm{~cm}^{-2}$.

The fluence dependence of $\mathrm{V}_{\text {depl }}$ for pions was measured by several groups. The ECP and Do groups measured on single detectors directly in the breaks between subsequent fluence steps, whereas the PPE data was taken for each fluence point on an individual detector after one week of annealing at room temperature and three months at $0{ }^{\circ} \mathrm{C}$. In all cases, $\mathrm{V}_{\mathrm{depl}}$ decreases first, reaches very small values and increases for fluences larger than approximately $10^{13} \mathrm{~cm}^{-2}$. This behaviour has also been seen for neutrons and protons and is related to an inversion of the bulk doping from n- to p-type silicon [6], [11].

Figure 7 shows the change of $\mathrm{N}_{\mathrm{eff}}$ versus the pion fluence $(350 \mathrm{MeV} / c)$ and the fits carried out according to Eq. (7).

Since the parameter $\mathrm{c}$ is strongly dependent on the starting material and difficult to determine out of the few existing data points, it is more advisable to compare the acceptor creation rate $b$ for pions with those values obtained for protons as listed in Table 4.

The values of $b$ are the same within a factor of two for all detector types and also compared with the values obtained for $24 \mathrm{GeV} / c$ protons and $1 \mathrm{MeV}$ neutrons. A closer agreement of these values cannot be expected since they do not include any annealing corrections.

In order to give a comparison of the long-term reverse annealing for different particles while excluding the variation due to different detector bulk material and production parameters, a dedicated experiment was carried out by the Hamburg group. Detectors were taken from only one wafer (HH1 type, $\mathrm{d}=329 \mu \mathrm{m}, \mathrm{N}_{\mathrm{eff}, 0}=4.5 \times 10^{11} \mathrm{~cm}^{-3}$ ), divided into three groups and exposed to neutrons, protons, and pions.

Neutron, proton and pion fluences for this experiment are all normalized to $1 \mathrm{MeV}$ neutron equivalent fluences, taking into account the non-ionizing energy loss and the energy spectra of the particle sources [6] (see Table 5). 


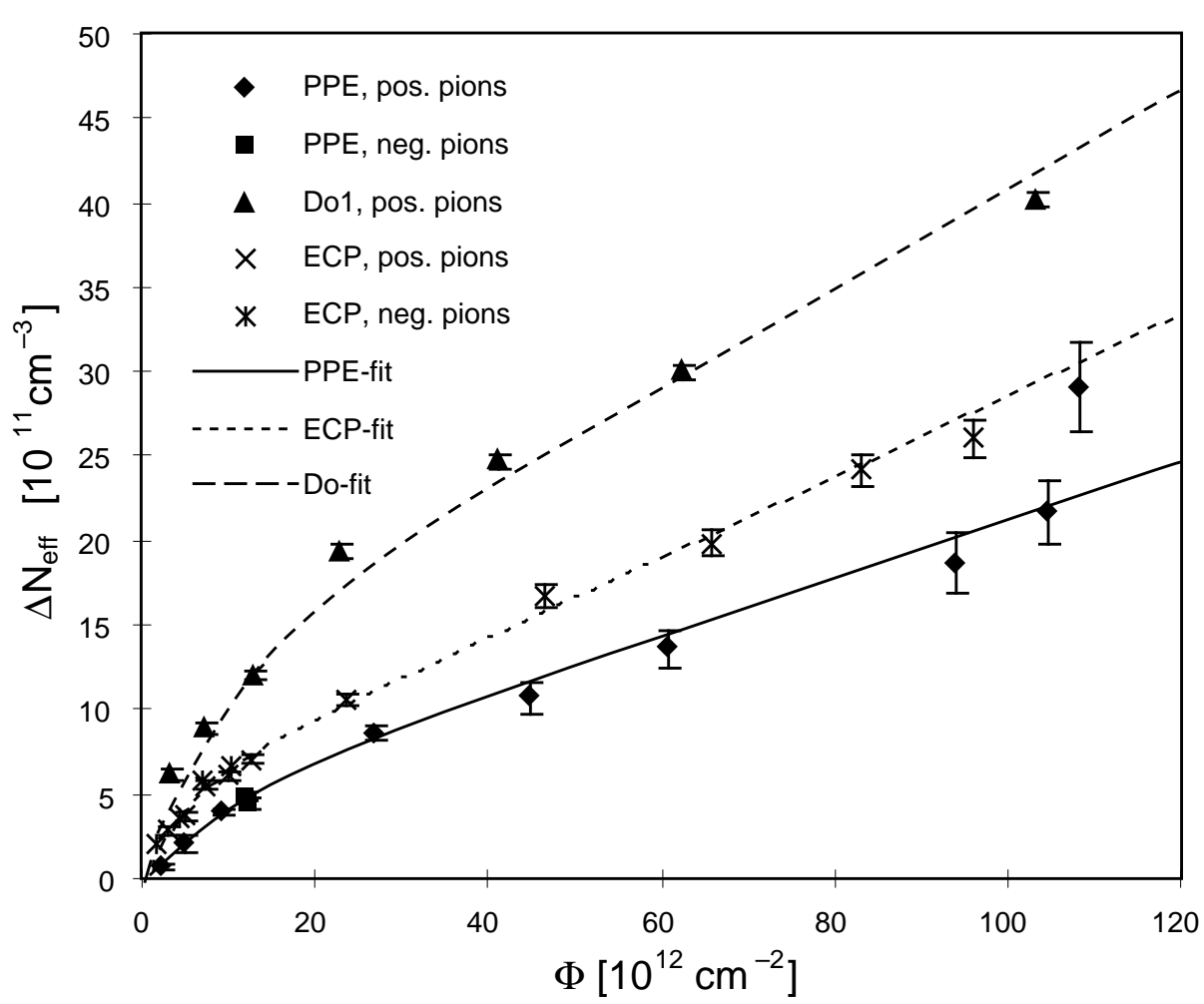

Fig. 7: Change of $\mathrm{N}_{\text {eff }}$ versus the pion fluence $(350 \mathrm{MeV} / c)$. Fits according to Eq. (7). ECP-fit only on data for positive pions, PPE fit including all data points. The ECP and Do-data were measured directly after irradiation, the PPE data after one week of room temperature and three months of $0{ }^{\circ} \mathrm{C}$ annealing.

Table 4

Acceptor creation rate $\mathrm{b}$ for the different pion-irradiated detector types and for proton- and neutron-irradiated detectors.

\begin{tabular}{|l|c|}
\hline \multicolumn{1}{|c|}{ Detector type, particle type } & $\mathrm{b}\left(10^{-2} / \mathrm{cm}\right)$ \\
\hline PPE, pos. and neg. pions $(350 \mathrm{MeV} / c)$ & $1.7 \pm 0.2$ \\
ECP, pos. pions $(350 \mathrm{MeV} / c)$ & $2.4 \pm 0.9$ \\
Do1, pos. pions $(350 \mathrm{MeV} / c)$ & $3.0 \pm 0.2$ \\
\hline protons $(24 \mathrm{GeV} / c)[11]$ & $2.8 \pm 0.1$ \\
\hline neutrons $(1 \mathrm{MeV})[13]$ & $3.1 \pm 0.2$ \\
\hline
\end{tabular}

Table 5

Radiation sources used for the direct comparison of long-term damage constants. The hardness factork is the normalization factor resulting from NIEL as function of particle energy and of the energy spectrum of the source $\left(\Phi_{\text {eq, }, 1 \mathrm{MeV}}=\kappa \times \Phi\right)$.

\begin{tabular}{|l|l|l|c|c|c|}
\hline \multicolumn{1}{|c|}{ Source } & Particle & \multicolumn{1}{|c|}{ Spectrum } & $\begin{array}{c}\text { (Mean) energy } \\
(\mathrm{MeV})\end{array}$ & $\begin{array}{c}\text { Ref. for } \\
\text { NIEL data }\end{array}$ & $\kappa$ \\
\hline PTB Be(d,n) & Neutron & Ref. [14] & 6.2 & {$[15]$} & 1.53 \\
CERN PS & Proton & Monoenerg. & 24000 & {$[16]$} & 0.93 \\
PSI $\pi$ E1 & Pion & Monoenerg. & 236 & {$[3]$} & 0.93 \\
\hline
\end{tabular}


The model applied for long-term annealing has been described elsewhere [17], [18]. The radiation-induced change of the effective impurity concentration as a function of fluence $\Phi$, time $\mathrm{t}$ and temperature $\mathrm{T}$ is given by:

$$
\Delta \mathrm{N}_{\mathrm{eff}}(\Phi, \mathrm{t}, \mathrm{T})=\mathrm{N}_{\mathrm{C}}(\Phi)+\mathrm{N}_{\mathrm{Y}}(\Phi, \mathrm{t}, \mathrm{T})
$$

where the term for the stable damage

$$
\mathrm{N}_{\mathrm{C}}(\Phi)=\mathrm{N}_{\mathrm{C} 0}\left(1-\mathrm{e}^{-\mathrm{c} \Phi}\right)+\mathrm{g}_{\mathrm{C}} \times \Phi
$$

has the form of fluence dependence already described in this section, but has to be evaluated at a point of time where only the stable fraction of the defects exists. This situation is reached after approximately one week of room-temperature annealing [6], therefore the $\mathrm{g}_{\mathrm{C}}$-value should be comparable to $\mathrm{b}$ out of the PPE measurement quoted above since the $0{ }^{\circ} \mathrm{C}$ storage avoids the onset of the long-term annealing $\mathrm{N}_{\mathrm{Y}}(\Phi, \mathrm{t}, \mathrm{T})$.

This long-term annealing is well described by second-order defect dynamics, which leads to [17], [18]

$$
\mathrm{N}_{\mathrm{Y}}(\Phi, \mathrm{t}, \mathrm{T})=\mathrm{g}_{\mathrm{Y}} \times \Phi \times\left(1-\frac{1}{1+\mathrm{g}_{\mathrm{Y}} \times \Phi \times \mathrm{k}(\mathrm{T}) \times \mathrm{t}}\right)
$$

where $\mathrm{k}(\mathrm{T})$ is a rate constant governed by a single activation energy. The generation rate gy can be taken from the $\mathrm{N}_{\text {eff }}$ saturation value. Figure 8 shows one example for the room-temperature annealing of $\Delta \mathrm{N}_{\mathrm{eff}}$ for a pion-irradiated detector. The different contributions to the annealing are indicated. Saturation is not reached after 200 days of annealing. The time until saturation can be shortened by heating the detector in the annealing phase. The saturation value and therefore $g_{Y}$ is not affected by this procedure, which was applied to all detectors in the following analysis.

Table 6 presents the results for the damage constants and the corresponding errors. If the NIEL hypothesis, i.e. the assumption that the damage is only scaled by the non-ionizing energy loss independent of the particle type, holds and if all energy spectra information is correct, the quoted values should be the same for all particle types. For both relevant parameters, $\mathrm{g}_{\mathrm{C}}$ and $\mathrm{g}_{\mathrm{Y}}$, this is true for neutrons and pions, whereas the proton values are smaller. There are many possible origins for this deviation: systematic errors in the fluence determination, errors in the NIEL calculation for protons resulting in too large a value for the hardness factor $\kappa_{\text {proton }}$, and the non-validity of the NIEL hypothesis itself. The latter explanation seems unlikely since, if there were discrepancies due to different particle interactions, one would more likely expect two different values for neutral (neutrons) and charged (protons, pions) particles.

The comparison with data from fits over many different detector types is also included in Table 6 . The values are of the same order, the discrepancies occur since in the global survey several radiation sources were included and the corresponding normalization to $1 \mathrm{MeV}$ neutron fluences is still a point of discussion. The $\mathrm{N}_{\mathrm{C} 0}$ values depend on the initial doping concentration. Thus, the data cannot necessarily be compared in this context. 


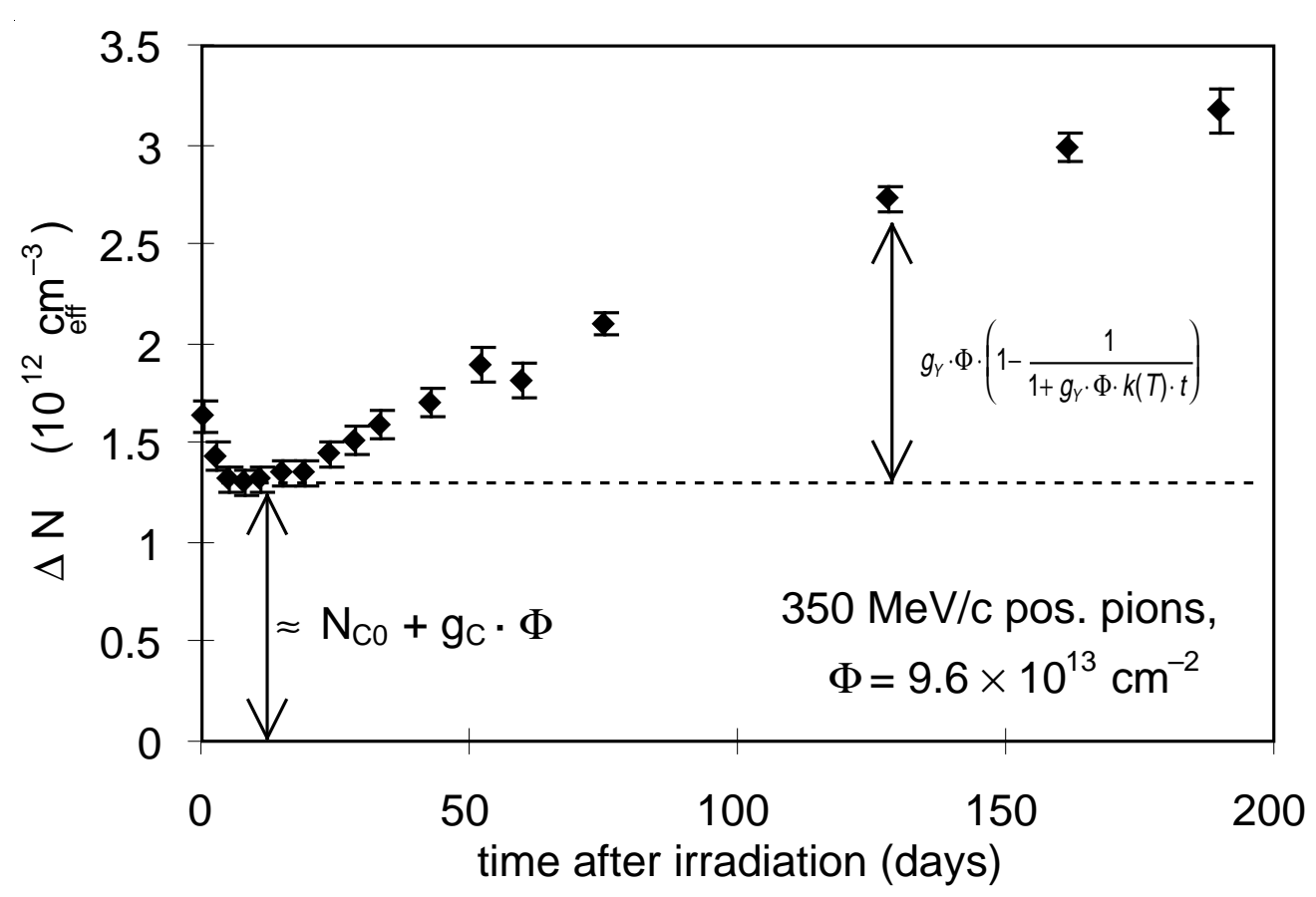

Fig. 8: Room-temperature annealing of $\Delta \mathrm{N}_{\text {eff }}$ for a highly irradiated detector (positive pions, $350 \mathrm{MeV} / c$, $9.6 \times 10^{13} \mathrm{~cm}^{-2}, \mathrm{~N}_{\mathrm{eff}}(\Phi=0)=4.8 \times 10^{11} \mathrm{~cm}^{-3}$, ECP type $)$.

Table 6

$1 \mathrm{MeV}$ equivalent damage constants and corresponding statistical errors. Data depends on the hardness factorsא (see Table 5). For comparison, the neutron and proton data from a global survey [1] and the corresponding standard-deviation error describing the spread are given.

\begin{tabular}{|l|c|c|c|c||c|c|}
\cline { 2 - 7 } \multicolumn{1}{c|}{} & $\begin{array}{c}\text { Neutrons, } \\
\text { this study }\end{array}$ & $\begin{array}{c}\text { Protons, } \\
\text { this study }\end{array}$ & $\begin{array}{c}\text { Pions } \\
\text { this study }\end{array}$ & $\begin{array}{c}\text { Pions, PPE } \\
\text { b value, one } \\
\text { week RT } \\
\text { annealing }\end{array}$ & $\begin{array}{c}\text { Neutrons, } \\
\text { global survey }\end{array}$ & $\begin{array}{c}\text { Protons, } \\
\text { global } \\
\text { survey }\end{array}$ \\
\hline $\mathrm{N}_{\mathrm{C} 0}\left[10^{12} \mathrm{~cm}^{-3}\right]$ & $\begin{array}{c}0.16 \pm 0.1 \\
3\end{array}$ & $\begin{array}{c}0.06 \pm 0.0 \\
5\end{array}$ & $\begin{array}{c}0.24 \pm 0.1 \\
5\end{array}$ & $0.42 \pm 0.11$ & $0.06 \ldots 0.33$ & $0.4 \ldots 0.7$ \\
$\mathrm{~g}_{\mathrm{C}}\left[10^{-2} \mathrm{~cm}^{-1}\right]$ & $2.5 \pm 0.3$ & $1.5 \pm 0.1$ & $2.5 \pm 0.3$ & $1.7 \pm 0.2$ & $1.8 \pm 0.4$ & $1.2 \pm 0.6$ \\
$\mathrm{~g}_{\mathrm{Y}}\left[10^{-2} \mathrm{~cm}^{-1}\right]$ & $5.9 \pm 0.3$ & $4.3 \pm 0.1$ & $6.3 \pm 0.7$ & & $4.6 \pm 1.6$ & $5.8 \pm 1.4$ \\
\hline $\begin{array}{l}\text { Number of detectors } \\
\text { irradiated }\end{array}$ & 5 & 4 & 8 & & & \\
\hline
\end{tabular}

\section{CONCLUSION}

The pion-induced damage in silicon detectors has been studied in the pion energy region of 65-330 MeV by different subgroups of the RD2 Collaboration in order to compare it with the results for neutron-induced damage and to check the non-ionizing energy loss predictions by Aarnio and Huhtinen.

The energy dependence of the leakage current increase agrees with the calculations for the energy dependence of the non-ionizing energy loss. There is no more enhanced damage at the $\Delta$ resonance than predicted by the NIEL calculations. The reverse current increase, the change 
in $\mathrm{N}_{\mathrm{eff}}$, and the charge collection efficiency deterioration due to $240 \mathrm{MeV}$ pions is, within the errors, the same as for $1 \mathrm{MeV}$ neutrons. The direct comparison of long-term annealing, scaling all damage constants to $1 \mathrm{MeV}$ neutron equivalent fluences, shows agreement of pion and neutron damage. Data from different detector types, which were entirely produced from similar bulk material but using different processes, show no deviations from each other.

Care has to be taken when considering surface effects leading to enhanced current damage after high fluences, which show a time dependence that is not well understood. However, these effects do not seem to be pion-specific and have also been observed for other particle types.

The study has shown that the pion-induced damage can be calculated to a good accuracy from $1 \mathrm{MeV}$ neutron data using the NIEL calculations. This means that the well-established results for $1 \mathrm{MeV}$ neutrons can be taken for predictions on irradiation-induced bulk damage of silicon detectors in high-energy physics experiments.

\section{ACKNOWLEDGEMENTS}

The authors would like to thank R. Horisberger, K. Gabathuler, and R. Frosch of the Paul Scherrer Institute for their help in providing beam time, beam diagnostic information, and beam setup. This work has been supported by the Bundesministerium für Bildung, Wissenschaft, Forschung und Technologie (BMBF) under the contract numbers $056 \mathrm{HH} 17 \mathrm{P}$ and 05 6DO52I.

\section{REFERENCES}

[1] A. Chilingarov et al., Nucl. Instr. and Meth. A360 (1995) 432.

[2] G. Gorfine and G. Taylor, CERN ATLAS-INDET-NO-030 (1993).

[3] M. Huhtinen and P.A. Aarnio, Nucl. Instr. and Meth. A336 (1993) 98.

[4] C. Furetta et al., CERN-ECP/95-2 (1995) and submitted to the Proceedings of the 4th Conference on Advanced Technology and Particle Physics, Villa Olmo, Como, October 1994.

[5] E. Léon-Florián et al., CERN-ECP/95-15 (1995).

[6] R. Wunstorf, PhD thesis, University of Hamburg, DESY FH1K-92-01 (October 1992).

[7] G. Lindström, 'Radiation damage in silicon detectors - self annealing corrections', SITP Internal Note, SITP-002 (1991).

[8] RD2 Collaboration, Nucl. Instr. and Meth. A326 (1993) 365.

[9] E. Fretwurst et al., Nucl. Instr. and Meth. A326 (1993) 357.

[10] S.J. Bates et al., 'Proton irradiation of silicon detectors with different resistivities', presented at Radiations et leurs effets sur les composants et systèmes (RADECS 95), Arcachon, September 1995; CERN-ECP/95-18 (1995).

[11] F. Lemeilleur et al., RD2 Collaboration, IEEE Trans. Nucl. Sci. 41 (1994) 425;

C. Leroy et al., RD2 Collaboration, CERN-ECP/93-12 (1993) and in Proceedings of the 4th International Conference on Calorimetry in High Energy Physics, Elba, Italy, 1993 (World Scientific, Singapore, 1994).

[12] A. Chilingarov et al., Nucl. Instr. and Meth. A337 (1993) 57. 
[13] S.J. Bates, Dissertation, University of Cambridge, RALT-006 (1993).

[14] H.J. Brede et al., Nucl. Instr. and Meth. A274 (1989) 332.

[15] M.S. Lazo et al., [Tech. Rep.] SAND, SAND87-0098 Vol. 1.

[16] A. van Ginneken, Technical Report FN-522, Fermi National Accelerator Laboratory (1989).

[17] E. Fretwurst et al., Nucl. Instr. and Meth. A342 (1994) 119.

[18] T. Schulz et al., IEEE Trans. Nucl. Sci. 41 (1994) 791. 